\title{
Inputs alter coupling strength
}

\section{cat \\ electrical \\ stimulation of glutamatergic input strengthened coupling between weakly coupled neurons}

Neurons in the inferior olive of the cerebellum are electrically coupled through gap junctions on dendritic spines. They also receive input from excitatory and inhibitory neurons. GABAergic neurotransmission is known to inhibit electrical transmission in the inferior olive, but it has been unclear whether excitatory input also influences electrical transmission. Three new studies now show that both GABAergic and glutamatergic projections can modulate the strength of electrical coupling between inferior olive neurons and alter neuron synchronization.

Mathy et al. and Turecek et al. performed patch-clamp recordings from coupled inferior olive neurons in rat brain slices. Mathy et al. found that $1 \mathrm{~Hz}$ electrical stimulation of excitatory input reduced the coupling strength between neuron pairs (assessed by calculating the coupling coefficient) for over 15 minutes. Importantly, the strength of excitatory chemical synapses in inferior olive neurons was not affected by stimulating glutamatergic input.

Inferior olive neurons show spontaneous 'subthreshold' oscillations in membrane potential, which can occur in the absence of action potentials. Turecek et al. showed that application of NMDA amplified and synchronized or induced synchronized subthreshold oscillations among neuron pairs - suggesting an increase in coupling. Interestingly, some NMDA receptors were extrasynaptic and neighboured gap junctions. Further experiments revealed that NMDA treatment increased the coupling coefficient between neurons that were initially weakly coupled, which were mainly non-neighbouring neurons. Like NMDA application, $9 \mathrm{~Hz}$ electrical stimulation of glutamatergic input strengthened coupling between weakly coupled neurons, an effect specifically related to NMDA receptor activation.

Mathy et al. and Turecek et al. further showed that the effects of excitatory input on coupling strength could be prevented by pretreatment with an NMDA receptor antagonist, a calcium chelator or a calcium/ calmodulin-dependent protein kinase II (CaMKII) inhibitor. Rapid calcium imaging by Turecek et al. also revealed that NMDA-induced strengthening of coupling and synchronization was associated with calcium influx near dendritic spines. Together, these findings indicate that changes in the strength of electrical coupling might depend on the frequency of excitatory stimulation and are mediated by a mechanism that involves calcium entry through NMDA receptors near gap junctions and CaMKII activation.

Lefler et al. focused on the effects of inhibitory input from the cerebellar nucleus on electrical coupling in the inferior olive. They optogenetically stimulated synaptic terminals of GABAergic nucleo-olivary axons and recorded activity in two inferior olive neurons simultaneously in mouse brain slices. Light-induced stimulation of nucleo-olivary input transiently reduced the strength of coupling between two inferior olive cells. The authors also showed that the coupling between a pair of neurons could be asymmetric and that nucleo-olivary input increased this asymmetry in some neuron pairs and decreased it in others, thereby modulating the preferred direction of current flow. Moreover, lightinduced stimulation of nucleo-olivary axons suppressed subthreshold oscillations. Thus, nucleo-olivary projections transiently reduce both coupling between inferior olive neurons and subthreshold oscillations in the inferior olive.

These studies show that the coupling strength of electrical synapses in the inferior olive can be modulated by synaptic input, with the direction and duration of the modulation depending on the type and frequency of the input. Electrical synapses also occur in other brain areas, and their modulation by chemical synapses is therefore likely to influence synchronization in many brain networks.

Leonie Welberg

ORIGINAL RESEARCH PAPERS Mathy, A., Clark, B. A. \& Häusser, M. Synaptically induced long-term modulation of electrical coupling in the inferior olive. Neuron 81, 1290-1296 (2014) | Turecek, J. et al. NMDA receptor activation strengthens weak electrical coupling in mammalian brain. Neuron 81, 1375-1388 (2014) | Lefler, Y., Yarom, Y. \& Uusisaari, M. Y. Cerebellar inhibitory input to the inferior olive decreases electrical coupling and blocks subthreshold oscillations. Neuron $\mathbf{8 1}$, 1389-1400 (2014)

FURTHER READING Pereda, A. E. Electrical synapses and their functional interactions with chemical synapses. Nature Rev. Neurosci. 15, 250-263 (2014) 